\title{
iSupport como herramienta modelo para el acceso a la justicia en el ámbito del derecho internacional privado
}

\author{
iSupport as a model tool to provide access to justice in the field of \\ private international law
}

\author{
Santiago Theoduloz \\ ORCID: 0000-0002-3316-5712 \\ Universidad de la República \\ Correo: santheoduar@gmail.com \\ Candela Villegas \\ ORCID: 0000-0001-5258-7091 \\ UNC-CONICET \\ Correo: candela_villegas@hotmail.com
}

Recibido: 16/07/2021

Aceptado: 03/11/2021

Resumen: El presente trabajo tiene como objetivo analizar la incorporación de la herramienta iSupport para mejorar el acceso justicia en los casos iusprivatistas que versen sobre obligaciones alimentarias transfronterizas y el apoyo que significa a las actividades que realizan las autoridades centrales de cada jurisdicción. Este mecanismo creado bajo el Reglamento de la Unión Europea sobre obligaciones alimentarias de 2009 y la Conferencia de La Haya en el Convenio sobre cobro Internacional de Alimentos para Niños y otros miembros de la Familia del 2007, pretende lograr mayor celeridad y disminución de costos en las tramitaciones de cobros internacionales de alimentos, por lo que se examinarán las ventajas y desventajas de este mecanismo y la capacidad de adaptación del Derecho internacional privado frente a los avances tecnológicos.

Palabras clave: obligaciones alimentarias; Isupport; autoridades centrales; avances tecnológicos; acceso a la justicia.

Abstract: The purpose of this paper is to analyze the incorporation of the iSupport tool to improve access to justice in justiciable cases involving cross-border maintenance obligations and the contribution it makes to the activities carried out by the central authorities of each jurisdiction. This mechanism, created under the European Union Regulation on maintenance obligations of 2009 and the Hague Conference on International Recovery of Child Support and Other Forms of Family Maintenance of 2007, aims to achieve greater celerity and lower costs in the processing of international maintenance claims. The advantages and disadvantages of this mechanism and the capacity of private international law to adapt to technological advances will be therefore examined.

Keywords: maintenance obligations; Isupport; central authorities; technological developments; access to justice. 


\section{Introducción}

Con el paso del tiempo, el derecho internacional privado (DIPr) ha sufrido frecuentes deformaciones formalistas. Estas deformaciones se explican por una visión excesivamente abstracta y conceptual de su función. Sin embargo, la funcionalidad del DIPr, como explica Dreyzin de Klor, es la de "alcanzar una solución justa en relación con el objeto que le compete", teniendo el carácter de un derecho facilitador del tráfico privado externo, y no un derecho directivo (Dreyzin de Klor, 2015, p. 35).

Así, uno de los principios del DIPr que se consagra como axioma rector de la materia, es la tutela judicial efectiva. Este principio, para su concreción, requiere del efectivo acceso a la justicia de los individuos y, consecuentemente, queda plasmado en otro principio como es el de no denegación de la justicia. En esta línea, la búsqueda de mecanismos que logren, de una u otra forma, llevar a la práctica los derechos y reglas adoptadas en los distintos instrumentos internacionales suponen, no solo una garantía de realización de los derechos allí consagrados, sino una forma de evolución del DIPr, haciendo más accesible a la justicia en el ámbito transnacional.

De esta forma, el efectivo acceso a la justicia puede afectarse por demoras y altos costos que impactan en los justiciables que se pueden ver impedidos de una real realización de sus derechos. Esta situación es contraria a los principios subyacente al DIPr, que buscan ser garantizados mediante los diversos instrumentos internacionales existentes, encontrándose en constante evolución.

El DIPr, en su carácter dinámico y flexible, debe administrar las herramientas necesarias para evitar situaciones que vulneren el efectivo acceso a la justicia de los individuos. Así, el uso e incorporación de distintas tecnologías es una herramienta útil para evitar situaciones de denegación internacional de justicia a causa de las distintas barreras que pueden existir entre los Estados. Sin dudas, los avances tecnológicos tienen un importante rol en el desarrollo del DIPr y todos los actores involucrados deben abogar por un uso efectivo de las herramientas que la tecnología brinda. Con un nuevo desafío - no menor- como es mantener las garantías de los justiciables, pero al mismo tiempo otorgar soluciones que siempre permitan una tutela efectiva de sus derechos.

El impacto que el uso de herramientas tecnológicas tiene en el avance del derecho es claramente primordial, pero dicha evolución debe transitar un camino de perfeccionamiento que determine la existencia de herramientas útiles y garantistas. No obstante, algunos procesos pueden llegar a suponer la necesidad de otorgar herramientas 
más rápidas en búsqueda de soluciones efectivas. De esta forma, no se puede dejar de mencionar el impacto que la pandemia causada por el virus COVID-19 ha supuesto en el desarrollo de diversas áreas, entre ellas el derecho. A finales de 2019 no se hubiera imaginado o pensado, por ejemplo, la realización de audiencias en formato totalmente virtual o la imposibilidad de viajar de una jurisdicción a otra y la necesidad de buscar soluciones tecnológicas para suplantar estas situaciones.

Lo cierto es que esta reciente y dinámica transformación del derecho, o de las herramientas de acceso a la justicia, tiene una base en desarrollos previos que en cierta medida sirvieron para una evolución necesaria y que han permitido, en una situación excepcional, velar por garantizar los principios más básicos de la disciplina.

Entre las herramientas tecnológicas que hacen al derecho y su evolución surge iSupport. Se trata de un mecanismo creado bajo el Reglamento de la Unión Europea (UE) sobre obligaciones alimentarias de 2009 y la Conferencia de La Haya sobre Cobro Internacional de Alimentos para los Niños y otros Miembros de la Familia de 2007. Es decir, en un ámbito en el cual la búsqueda de celeridad es de máxima necesidad y donde el justiciable no puede quedar como víctima de un sistema lento y costoso, cuando necesita de un sistema judicial que lo ampare y proteja. De esta forma, iSupport se convierte en una importante herramienta de apoyo a las distintas actividades que realizan las autoridades centrales de cada jurisdicción y tiende justamente a facilitar tal mecanismo.

El objetivo de este trabajo es presentar las principales características de iSupport en un marco de creciente evolución tecnológica, destacar su naturaleza como mecanismo facilitador de la realización de los distintos derechos consagrados a nivel internacional en concordancia con la evolución del DIPr y sus principios más básicos. Esto lleva a desarrollar en primer lugar las principales características del iSupport, para luego detenerse en la necesidad de buscar un eficaz acceso a la justicia a través de la tecnología, analizando los distintos campos en los que se pueden aplicar tecnologías como iSupport. 


\section{iSupport: un nuevo mecanismo al servicio del DIPr y la concreción de sus principios}

iSupport es un mecanismo que surge en el marco de la Conferencia de La Haya de Derecho Internacional Privado (en adelante $\mathrm{HCCH}$, por sus siglas en inglés). Se trata de un software que tiene como objetivo principal colaborar en hacer más eficaz y ágil el cumplimiento trasnacional de obligaciones alimentarias, evitando los costos que las transacciones bancarias suponen, así como los perjuicios que las demoras en su ejecución puede suponer para las personas involucradas.

iSupport se presenta entonces como un sistema eficaz en el manejo y comunicación de aquellos casos trasnacionales de obligaciones alimentarias y se inserta -conforme se indicará supra- en el marco del Reglamento CE 4/2009 del Consejo de la Unión Europea del 18 de diciembre de 2008 relativo a la competencia, la ley aplicable, el reconocimiento y la ejecución de las resoluciones y la cooperación en materia de obligaciones de alimentos, así como en la Convención de La Haya el Convenio sobre cobro Internacional de Alimentos para Niños y otros miembros de la Familia del año 2007.

Tanto el reglamento como la convención en el uso de este tipo de mecanismos tienen el potencial de beneficiar a miles de niños y adultos en Europa y en todo el mundo, al facilitar el envío transfronterizo del pago de las correspondientes obligaciones alimentarias cuando se dan en el marco de un caso internacional. En relación con la relevancia de este tipo de herramientas, que hacen de base para el desarrollo propio de iSupport, se ha detectado que en países como Australia, Canadá, Nueva Zelanda y los Estados Unidos, en promedio, hay un caso de manutención infantil internacional por cada mil habitantes ( $\mathrm{HCCH}, 2009)$. Números que dejan ver claramente la necesidad de contar con un mecanismo que agilice y facilite la efectivización de las obligaciones alimentarias.

\section{Objetivos del sistema iSupport}

En el proyecto para el desarrollo de iSupport se describe que este tipo de instrumentos tienden a construir puentes a nivel internacional entre los diferentes sistemas legales nacionales en relación con las obligaciones alimentarias. Así, se hace énfasis en que un sistema de estas características podrá proporcionar un puente electrónico entre Estados interesados en comunicar, procesar datos y transferir pagos 
bajo este tipo de instrumentos internacionales. Todo ello en el marco de la cooperación jurídica internacional.

Esta cooperación es posible gracias a la migración electrónica de los datos requeridos en la convención y el Protocolo para el Desarrollo de Isupport. De esta manera, se busca asegurar que los instrumentos se utilicen en todo su potencial y faciliten la obtención de la obligación alimentaria correspondiente de una forma rápida, eficiente y rentable.

iSupport tiene como objetivo, mediante el uso de la tecnología y sus beneficios, ahorrar dinero, agilizar los trámites burocráticos y permitir un efectivo acceso a la justicia de las partes involucradas. El proyecto inicial contó con el financiamiento de la UE así como de algunos países miembros de la $\mathrm{HCCH}$ y organizaciones internacionales. En el 2016, se implementó el programa a través de pruebas pilotos en Portugal y en el estado de California, Estados Unidos.

Posteriormente la UE financió desde 2016 a 2018 otro proyecto denominado iSupport 2.0, con el objetivo de agregar y mejorar funcionalidades del software anterior, buscando además la incorporación de nuevos países en la utilización del sistema. En el período de setiembre 2018 a 2020, un nuevo proyecto también financiado por la UE y denominado iSupport PM (Promotion \& Maintenance) involucró a 15 países ${ }^{1}$ con el objetivo de continuar mejorando el sistema y lograr que participen la mayor cantidad de Estados posibles, lo que redunda en una mejora integral del sistema.

Cabe señalar que este mecanismo si bien se inserta en un área específica, las obligaciones alimentarias representan un ejemplo a seguir por parte de los Estados en la mejora del acceso a la justicia de quienes muchas veces ven vulnerados sus derechos por el simple hecho de vivir en Estados diferentes. Las condiciones están dadas para la extensión del uso de herramientas como iSupport a nivel de distintas ramas del derecho en base a principios generales que deberán considerarse dependiendo del área concreta.

Así, en materia de obligaciones alimentarias se encuentran problemas específicos, entre ellos, que las transferencias internacionales terminan redundando más en beneficio de las instituciones financieras que de los propios beneficiarios de las pensiones alimenticias. Situación que perjudica a quienes ya se encuentran en una situación de clara vulnerabilidad. Frente a estas situaciones, la utilización de este tipo de herramientas es sumamente beneficioso. Lo anterior sumado a que el hecho de lograr

\footnotetext{
${ }^{1}$ Alemania, Austria, Brasil, Eslovaquia, Estados Unidos, Estonia, Finlandia, Francia, Grecia, Hungría, Noruega, Países Bajos, Portugal, República Checa y Suiza (HCCH, Isupport).
} 
celeridad en este tipo de procedimientos puede en algunas situaciones resultar vital para los involucrados.

Es de destacar que, como han establecido los desarrolladores de iSupport, su uso favorece y facilita las comunicaciones entre las autoridades centrales. Contribuye, además, a la actividad de estas, mejorando los estándares de gestión y monitoreo de los casos. Esto se materializa en que iSupport opera en varios idiomas -facilitando así la traducción a diferentes idiomas- y permite tener acceso a estadísticas, que fueron desarrolladas como parte de los medios de vigilancia del funcionamiento en la convención.

\section{Procedimiento}

En relación con el procedimiento de esta herramienta, Maja Groff destaca que con iSupport se planea un nuevo sistema global de administración de casos para aprovechar soluciones tecnológicas, apoyando soluciones que tornen las obligaciones alimentarias en una práctica efectiva. Las autoridades centrales designadas en virtud de la convención tendrían así la capacidad de comunicarse entre sí de forma rápida y eficiente, para gestionar casos iusprivatistas en materia de alimentos, abordando satisfactoriamente las barreras idiomáticas que puedan presentarse, considerándose además la rapidez y el bajo costo de las transferencias involucradas. iSupport tiene sin dudas el potencial de ayudar a una aplicación más eficiente y estandarizada de la convención, ahorrando tiempo y dinero para autoridades nacionales y efectivamente obtener más dinero en manos de los acreedores en forma más rápida, lo que redunda en un beneficio para estos (Groff, 2014).

Como puede observarse en la imagen 1, el software se comunica con las autoridades centrales de los diferentes Estados involucrados mediante un sistema informático con datos encriptados que protege contra riesgos de pérdida, robo, daño y alteraciones no autorizadas.

Cuando se desarrolló iSupport, se planeó como objetivo central que dicho sistema acelerara las transferencias monetarias internacionales (con un esquema para reducir las tarifas de transferencia), disminuyendo los costos de transacción asociados con tales flujos internacionales de divisas. En el campo de las remesas de migrantes internacionales, se ha observado que los altos costos de transacción pueden disminuir los efectos positivos de estas transferencias transfronterizas y es, justamente, lo que se quiere impedir mediante el uso de iSupport. 


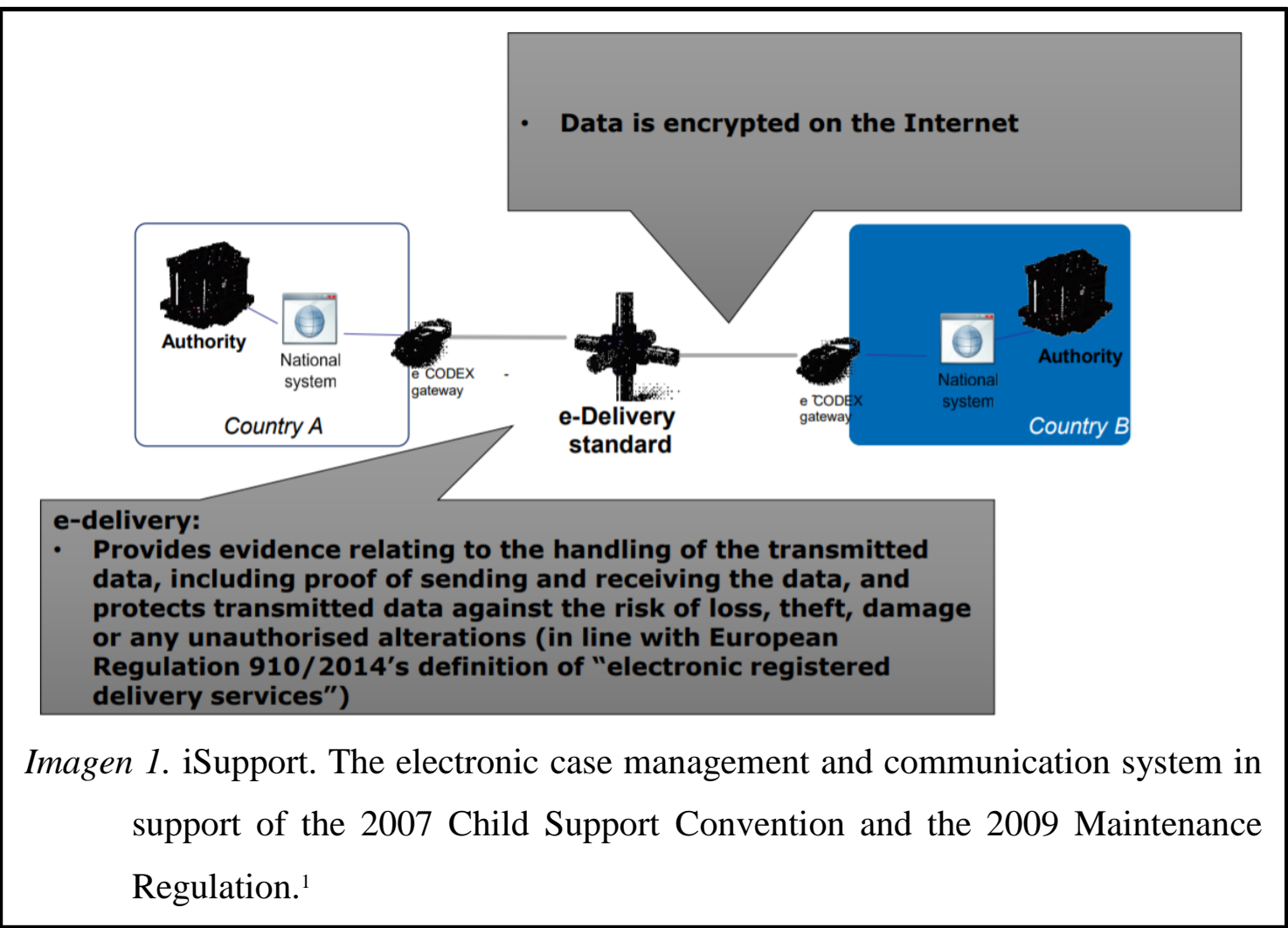

Asimismo, conforme surge de la imagen 1, iSupport utiliza como plataforma de comunicación el sistema e-Codex. Este sistema desarrollado en el marco de la UE tiene como objetivo crear un mecanismo seguro de comunicación a nivel de administración de justicia, a la vez que busca reducir los costos en la comunicación transfronteriza (eCodex) y del mismo se ha beneficiado iSupport. Conforme han señalado Burnett y Rongione (2019), desde que iSupport utiliza la plataforma e-Codex se ofrece un ámbito seguro para el intercambio electrónico de información de carácter sensible entre las autoridades centrales. En este sentido, esta sensibilidad es requerida dado el carácter de la información utilizada por iSupport. Por su parte, en relación especifica con el sistema iSupport, el reporte antes citado también hace referencia a que reduce el tiempo de comunicación entre las autoridades centrales, a la vez que supone la reducción de costos, así como de errores (Burnett \& Rongione, 2019).

En cuanto a este último aspecto y referido a la reducción de costos, es relevante considerar que iSupport utiliza la plataforma EULP, ${ }^{2}$ la Licencia Pública de la Unión Europea diseñada para el desarrollo de los programas. Así, la propia HCCH reconoce que la utilización de esta licencia pública le ha permitido a los Estados involucrados reducir

\footnotetext{
${ }^{2}$ La versión 1.2 de la licencia fue aprobada por la Decisión de Ejecución (UE) 2017/863 de la Comisión Europea del 18/05/2017 (Unión Europea, 2017, mayo 18).
} 
costos en forma considerable, a la vez de proveer a los ciudadanos de un acceso efectivo a la justicia (HCCH, Isupport).

\section{El acceso a la justicia a través de la tecnología}

En este apartado y en base a las líneas desarrolladas, se plantea analizar cuáles son los desafíos y ventajas que presenta una herramienta como iSupport, que se funda en la vital importancia que un mecanismo de este tipo supone para el progreso en materia de acceso a la justicia en el ámbito internacional. Esta idea implica entonces reconocer la relevante conexión existente entre un mayor y más fácil acceso a la justicia y el desarrollo de nuevas tecnologías. Es por eso que se cree que el acceso a la justicia a través de la tecnología es una forma fundamental para evitar que las fronteras funcionen como barreras para los justiciables, sobre todo para los más vulnerables. Máxime cuando es la existencia de fronteras jurisdiccionales lo que tiene como efecto que los costos aumenten, lo que supone que - en la mayoría de los casos- las posibilidades de acceso a la justicia disminuyan.

En el ámbito latinoamericano -sin perjuicio de su alcance global- los Principios de la Asociación Americana de Derecho Internacional Privado (Asadip) sobre el Acceso Transnacional a la Justicia (Transjus) son un importante elemento para considerar a la hora de desarrollar la temática objeto del presente. Estos principios ponen en el centro la necesidad de que existan ciertas garantías con el fin de acortar las diferencias que existen en el acceso a la justicia en el área del DIPr. Como indican los propios principios:

Establecen estándares mínimos para garantizar el acceso a la justicia, sin discriminación por razón de la nacionalidad o residencia y de conformidad con el Derecho internacional de los derechos humanos y los principios consagrados por la generalidad de las constituciones modernas (Asadip, Principios Transjus).

Destacándose a su vez que el acceso a un debido proceso es un DDHH, por lo que resulta de vital importancia establecer mecanismos que supongan su salvaguarda y garantía. Por cuanto, como también se destaca, estos principios "están inspirados y a la vez constituyen aplicaciones específicas de principios de ius cogens, en particular del derecho humano de acceso a la justicia" (Asadip, Principios Transjus). 
Naturalmente todos los principios que emanan de dicho documento tienen un fuerte impacto en el objeto de este estudio y tienden a garantizar el acceso a la justicia. Pero para este caso en particular, se destaca el principio de "máximo respeto de los derechos humanos y acceso a la justicia" y el principio de "cooperación jurídica internacional". En relación con este último punto, al tratar en su artículo 4.1 elementos estrictamente de cooperación jurisdiccional, menciona que:

La cooperación interjurisdiccional constituye una obligación internacional de todo Estado, no solamente en a los efectos de realizar emplazamientos, notificaciones y audiencias, sino que el deber de cooperar se extiende a todos aquellos actos o medidas necesarios para la consecución de los fines del proceso, incluyendo la cooperación en el procesamiento y ejecución de medidas cautelares, así como en la identificación, conservación y producción de pruebas (Asadip, Principios Transjus).

De acuerdo con el principio antes transcripto, los Principios Asadip van un paso más allá e indican en su art. 4.7 que:
Siempre que se garantice la seguridad de las comunicaciones, los jueces y demás operadores de justicia procurarán y favorecerán el uso de las nuevas tecnologías de información y comunicación, tales como comunicaciones telefónicas y videoconferencias, mensajes electrónicos, y cualquier otro medio de comunicación apto para hacer efectiva la cooperación solicitada (Asadip, Principios Transjus).

Por tanto, los propios Principios Asadip reconocen expresamente la importancia de la incorporación de elementos tecnológicos en el acceso de la justicia trasnacional, tal como es el uso de una herramienta del estilo de iSupport.

En el mismo sentido que estos principios -y a modo de ejemplo-el Protocolo Iberoamericano de Cooperación Jurídica Internacional, cuya aprobación fue realizada en la XVII Cumbre Judicial Iberoamericana, celebrada en el año 2014 en Santiago de Chile, también menciona la necesidad de promover el uso de la tecnología en materia de cooperación jurisdiccional y principalmente en la búsqueda de la eficacia de la función jurisdiccional (XVII Cumbre Judicial Iberoamericana, Declaración de Santiago de Chile). 
Es por ello que se afirma que la importancia de facilitar un mejor acceso a la justicia responde al hecho de que se trata de un DDHH fundamental que merece las máximas salvaguardas, así como la búsqueda de desarrollar las herramientas que lo garanticen, evitando las asimetrías que factores económicos pueden producir en los justiciables.

Así, en línea con lo expresado anteriormente se ha manifestado que:

Las empresas y personas con mayores recursos, encuentran en los avances actuales del Derecho internacional privado, herramientas más o menos eficaces para hacer valer sus derechos. Entre los mecanismos arbitrales y la sofisticada asistencia jurídica internacional, los derechos de éstas se encuentran relativamente bien tutelados. Pero las asimetrías entre las partes suelen incrementarse más en la esfera de las relaciones privadas internacionales. Las corporaciones y personas con más recursos, pueden acceder a servicios jurídicos altamente especializados, con agencias en múltiples jurisdicciones, lo que les permite planificar y ejecutar estrategias de litigio transnacionales, incoando múltiples procesos al mismo tiempo, evacuando pruebas en distintas jurisdicciones, ejecutando u oponiéndose medidas cautelares, etc. Tales asimetrías tienden a convertirse en desventajas insuperables para los más débiles en el litigio internacional. Y así, lo que para algunos son obstáculos infranqueables, resultan para otros las mejores herramientas para imponerse y prevalecer (Madrid \& Ochoa, 2014, p. 299).

Lo expresado anteriormente lleva justamente a reflexionar que un fin primordial del Estado de derecho debe ser el de evitar la existencia de asimetrías, que son generadas por los obstáculos que producen la existencia de fronteras y que muchas veces los propios Estados incrementan. Esto es, ante la imposición de requisitos que muchas veces se convierten en verdaderos obstáculos. Si bien los requisitos que los Estados disponen buscan garantizar una justicia efectiva, la búsqueda de garantías no puede suponer alejar a los justiciables de una justicia efectiva. Como en todos los órdenes, la búsqueda de un equilibrio entre garantías y acceso en la justicia debe ser el eje que guie el desarrollo de herramientas que incorporen el uso de tecnologías.

En cuanto a las asimetrías, la práctica actual indica, que aun con un rol más activo de las autoridades centrales y sus esfuerzos, el diligenciamiento de cualquier tipo de exhorto o carta rogatoria es más fácil si la persona que intenta acceder a la justicia tiene la posibilidad, de una u otra manera, de contar con profesionales de derecho en ambas 
jurisdicciones. Es sabido que dicha realidad muchas veces es a todas luces excepcional y esto no puede escapar a los actores jurídicos. La existencia de un mecanismo como iSupport, que tiene como fin principal el de acortar desigualdades en materia de acceso a la justicia se constituye en una herramienta a estudiar y principalmente a celebrar y promover.

Por ello se considera que todo esfuerzo en la búsqueda de herramientas basadas en tecnologías es bienvenido y debe realizarse en conjunto con todos los actores involucrados, puesto que el desarrollo de herramientas tecnológicas al servicio del DIPr involucra no solo a todos los operadores jurídicos, sino también a la función administrativa a través del trabajo de la autoridad central y/o la autoridad competente y la función jurisdiccional, llevada a cabo por la autoridad judicial de cada país.

Ahora, como expresan los autores antes citados: "la meta de una justicia substantiva y procesal en la vida privada internacional está aún lejos de ser alcanzada" (Madrid \& Ochoa, 2014, p. 301). Por ello, se entiende que hacer hincapié en la incorporación de nuevas tecnologías como iSupport beneficiará un objetivo común de los Estados, la promoción del acceso a la justicia de todos sus ciudadanos sin importar la jurisdicción en la que se encuentren. El acceso a la justicia debe pensarse junto al deber de cooperación judicial que existe entre los Estados, que como se vio, su consideración se impone a la hora de hablar de un derecho fundamental, como es el acceso a una justicia eficaz, libre, equitativa y garantista.

Respecto de lo anterior, a la hora de analizar la coexistencia y cohesión entre el derecho internacional, los derechos humanos y el activismo judicial se ha expresado que:

En lo que va del siglo XXI las situaciones privadas internacionales han aumentado en forma exponencial con importantes consecuencias de cara a la juridicidad. Las causas que confluyen para destacar este fenómeno son variadas y numerosas. Así, se ha reiterado hasta el cansancio la importancia que reviste en la dinámica jurídica contemporánea, el incremento de los medios de comunicación internacional, el desarrollo de la informática, así como una paralela flexibilización de las fronteras nacionales y la incidencia de variables políticas, sociales, culturales y económicas, en tanto factores que contribuyen por un lado, a dinamizar los desplazamientos transnacionales y por otro, a acceder al conocimiento 
de datos con efectos inmediatos en las personas y las sociedades (Dreyzin de Klor, 2012).

Se realza la importancia de la tecnología en el desarrollo del DIPr y, en definitiva, los DDHH y la influencia su uso y desarrollo en diversas variables. Debe considerarse que tradicionalmente se ha hecho hincapié en la importancia que presenta para el desarrollo del DIPr actual la existencia de las autoridades centrales, como institución con el rol primordial de llevar a cabo los objetivos desarrollados por las distintas convenciones internacionales existentes y adoptadas por los Estados. No obstante, se ha observado que la forma de trabajo actual de la mayoría de las autoridades centrales y sus comunicaciones no siempre suponen la celeridad, que es claramente requerida para el funcionamiento efectivo del DIPr. Allí es donde se ve con mayor claridad la importancia y necesidad de desarrollar mecanismos como iSupport en colaboración con las tareas que estas realizan. Como señala Tellechea Bergmann:

La celeridad en la realización de la justicia impone analizar la posibilidad del uso de medios electrónicos tanto para la transmisión de los exhortos, cuanto para otras comunicaciones entre las autoridades centrales referidas al cumplimiento de los mismos. Posibilidad que cuando estos organismos fueran creados en los años sesenta y setenta del siglo veinte por las Convenciones de La Haya e Interamericanas no era previsible, pero que hoy constituye una realidad que permite a través de actuales tecnologías una comunicación instantánea y segura, capaz de acreditar la autenticidad del pedido (2017, p. 110).

Una interpretación evolutiva de los elementos que emergen de las distintas convenciones existentes impone la necesidad de buscar incorporar las nuevas tecnologías como herramientas principales que faciliten el trabajo de las autoridades centrales. Esto implica, en definitiva, el uso de nuevas tecnologías como herramienta vital para alcanzar un mayor acceso a la justicia, más justo, equitativo y que evite las asimetrías que distintas situaciones económicas pueden producir, que los Estados no pueden permitir, siempre manteniendo el equilibrio entre garantías y efectividad. 


\section{iSupport y el impacto en el DIPr actual}

iSupport supone un relevante adelanto en el acceso a la justicia y en consecuencia una garantía de tutela judicial efectiva frente a casos de obligaciones alimentarias, que requieren de medidas actuales y ajustadas a las problemáticas específicas. El uso de este tipo de tecnología facilita la labor de las autoridades centrales y su utilización mejora su gestión interna, traduciéndose en economía de tiempos y costos. El sistema iSupport también supone la introducción de una herramienta informática innovadora para los Estados que aún no han adoptado las prácticas de gobierno electrónico en materia de cooperación jurídica internacional, incluso inspirándolos a incorporar a nivel interno este tipo de sistemas, así como para un mayor número de convenciones en las cuales ello sea viable.

Herramientas como iSupport se justifican en la necesidad de que existan nuevos mecanismos que permitan un mayor acceso a la justicia en cada vez más áreas, de forma de evitar que la desigualdad económica produzca desigualdad jurídica. La idea puede suponer una utopía inicial, pero el uso de las tecnologías permite al Estado abaratar costos y es un rol del Estado garantizar el acceso a la justicia.

Como destaca Maja Groff (2014), según la convención de 2007, los Estados están obligados a dar la máxima prioridad a la transferencia internacional de obligaciones alimentarias. El rol del Estado es preponderante, como lo ha expresado Albornoz:

El acceso a la justicia en las relaciones privadas internacionales es un tema que reviste suma importancia en un contexto de globalización como el actual. En él gravitan intereses de las personas involucradas y del Estado cuyos jueces podrían o deberían intervenir para solucionar una controversia concreta. El interés de las personas en ejercer el derecho fundamental de acceso a la justicia, como medio para satisfacer una pretensión sustantiva, converge con el interés del Estado en la medida en la que éste debe garantizar que los justiciables efectivamente accedan a la justicia. Pero a su vez, el Estado está interesado en limitar el ejercicio de su jurisdicción y en este punto puede haber una divergencia con el acceso a la justicia (2017, p. 191). 


\section{Consideraciones finales}

El escenario mundial globalizado, con el consecuente crecimiento de las herramientas tecnológicas, impacta en cada una de las ramas del derecho, y el DIPr no puede ser ajeno a este fenómeno, siendo un área que tiene una verdadera posibilidad de adaptarse a los tiempos venideros. Es hora de que el DIPr utilice las herramientas tecnológicas que se encuentran a su alcance para lograr su propio desarrollo y lograr mediante la cooperación jurídica internacional un acceso efectivo a la justicia, evitando que las distintas asimetrías -existentes entre los justiciables- afecten sus derechos sustanciales.

El derecho de acceso a la justicia, sin dudas, puede servirse de las herramientas que la tecnología pone a disposición para su efectivización, sorteando así -como en el caso de iSupport- las dificultades que plantea el cumplimiento de las obligaciones alimentarias $-\mathrm{u}$ de otro tipo en caso de corresponder- cuando las mismas son transfronterizas y encuentran distintos obstáculos para la concreción de los fines de las normas concretas.

En este marco, se entiende la relevancia de la existencia de mecanismos como iSupport, no solo porque supone una herramienta vital en el acceso a la justicia y a la realización de la justicia, sino porque justamente este mecanismo se desarrolló inicialmente en un ámbito de importancia vital, como es el de derechos de alimentos, buscando facilitar y acotar gastos para una categoría o rango de justiciables que muchas veces no cuenta con los recursos necesarios. Esto no supone que no deban desarrollarse estas herramientas en otras áreas, como pueden ser las relativas a los contratos comerciales internacionales y su ejecución, por lo cual se aboga. Pero es relevante iniciar por aquellas áreas en las que los justiciables son aún más vulnerables.

iSupport es una experiencia nueva que, si bien se encuentra en período de evaluación para detectar posibles mejoras a introducir, sin lugar a dudas se presenta como un camino viable a seguir por parte de los Estados en la búsqueda de un mejor acceso a la justicia. iSupport se constituye además en un mecanismo que podrá ser adoptado en otras áreas que también son relevantes para el desarrollo de los DDHH y el acceso a la justicia, para luego ser ejemplo en otras áreas del DIPr. Ello no quita la relevancia que este tipo de herramientas tienen como puntapié inicial para la transformación de las autoridades centrales del futuro y un respaldo para la búsqueda de una justicia cada vez más accesible a nivel trasnacional. 
La tecnología debe servir al derecho, de tal manera que este pueda beneficiar a la humanidad y así lograr cumplir con los cometidos naturales del derecho. Más allá de los textos de las convenciones, que establecen patrones claros en base a la concreción de principios propios del DIPr, debe buscarse la forma para que estos se concreten. Por ello, se vio la importancia de, por ejemplo, los Principios Asadip, donde los elementos señalados se cristalizan con la inclusión de tecnologías y en la búsqueda de un DIPr cada vez más eficiente.

Por último, cabe señalar que el rol de la tecnología al servicio del derecho ha crecido en importancia, si se consideran los desafíos que el mundo ha atravesado con la pandemia de coronavirus. Situación a la cual el DIPr no puede estar ajeno, y demuestra la necesidad de estar preparados para un futuro que -aun con avances tecnológicos nunca antes pensados- no deja de ser incierto.

\section{Referencias}

Albornoz, M. M. (2017). Acceso a la justicia en las relaciones privadas internacionales: intereses en juego. Revista de la Secretaría del Tribunal Permanente de Revisión, 9, 170-195. doi: https://doi.org/10.16890/rstpr.a5.n9.p170

Asociación Americana de Derecho Internacional Privado [Asadip]. (2016). Principios Asadip sobre el Acceso Trasnacional a la Justicia (Tranjus). Recuperado de http://www.asadip.org/v2/wp-content/uploads/2018/08/ASADIP-TRANSJUSES-FINAL18.pdf

Burnett, M. \& Rongione, C. (2019). New solutions to complex challenges: A public sector citizen-centric, sustainable and fit for the future. Holanda: European Institute of Public Administration. Recuperado de https://epsa2019.eu/files/EPSA2019_publication_web.pdf

Conferencia de La Haya de Derecho Internacional Privado [HCCH]. (2009). Draft Business Plan for the Development of isupport. The Electronic case Management and Communication System for The Hague Convention of 23 November 2007 on the International Recovery of Child Support and other forms of Family Maintenance and The Hague Protocol of 23 November 2007 on the Law Applicable to Maintenance Obligations. Recuperado de https://assets.hcch.net/docs/b928d737-821c-43bf-a756-e9bbf32e188c.pdf

Conferencia de La Haya de Derecho Internacional Privado [HCCH]. Isupport. Recuperado de https://www.hcch.net/en/instruments/conventions/specialisedsections/child-support/isupport1

Dreyzin de Klor, A. (2012). Derechos humanos, derecho internacional privado y activismo judicial. XXXIX Curso de Derecho Internacional OEA, 1-22. 
Dreyzin de Klor, A. (2015). El Derecho Internacional Privado actual (Tomo 1). Buenos Aires: Zavalía.

e-Codex. About. Recuperado de https://www.e-codex.eu/about

Groff, M. (2014). Global Poverty and Economic Rights: the potential of the 2007 Child Support Convention. The Recovery of Maintenance in the EU and Worldwide Studies in Private International Law.

Madrid, C. \& Ochoa, J. (2014). Problemas de acceso trasnacional a la justicia en el derecho internacional privado. Perspectiva latinoamericana. XLI Curso de Derecho Internacional OEA, 281-346.

Tellechea Bergmann, E. (2017). Hacia una necesaria profundización de la cooperación jurisdiccional internacional y el reconocimiento de los fallos extranjeros en el ámbito interamericano. Revista de Derecho de la Universidad de Montevideo, 32 .

XVII Cumbre Judicial Iberoamericana; Declaración de Santiago de Chile. Disponible en: https://www.pj.gov.py/descargas/ID1-543_declaracion_santiago_chile.pdf

\section{Normativas}

Conferencia de La Haya de Derecho Internacional Privado [HCCH]. (2007). Convenio sobre cobro Internacional de Alimentos para Niños y otros miembros de la Familia. Recuperado de https://assets.hcch.net/upload/finact21s.pdf

Unión Europea [UE]. (2009). Obligaciones de alimentos. Recuperado de https://eurlex.europa.eu/legal-content/ES/LSU/?uri=CELEX\%3A32009R0004

Unión Europea [UE]. (2008, diciembre 18). Reglamento (CE) 4/2009 del Consejo. relativo a la competencia, la ley aplicable, el reconocimiento y la ejecución de las resoluciones y la cooperación en materia de obligaciones de alimentos. Recuperado de https://eur-lex.europa.eu/legalcontent/ES/TXT/PDF/?uri=CELEX:32009R0004\&from=EN

Unión Europea [UE]. (2017, mayo 18). Decisión de Ejecución (UE) 2017/863 de la Comisión. Por la que se actualiza la licencia EUPL de los programas informáticos de fuente abierta para seguir facilitando el intercambio y la reutilización de los programas desarrollados por las administraciones públicas. Recuperado de https://joinup.ec.europa.eu/sites/default/files/custompage/attachment/eupl_v1.2_es.pdf

\section{Contribución autoral}

a) Concepción y diseño del trabajo; b) Adquisición de datos; c) Análisis e interpretación de datos; d) Redacción del manuscrito; e) revisión crítica del manuscrito.

S. T. ha contribuido en a, b, c, d, e; C. V. en a, b, c, d, e.

Editora científica responsable: Dra. María Paula Garat. 